\title{
Detection Limits of Electron and Electron Capture Negative Ionization-Mass Spectrometry for Aldehydes Derivatized with $o-(2,3,4,5,6-$ Pentafluorobenzyl)-Hydroxylamine Hydrochloride
}

\author{
Josef Beránek, ${ }^{a}$ Darrin A. Muggli, ${ }^{b}$ and Alena Kubátováa \\ a Department of Chemistry, University of North Dakota, Grand Forks, North Dakota, USA \\ b Department of Chemical Engineering, University of North Dakota, Grand Forks, North Dakota, USA
}

In contrast to common expectations, the differences in limits of detection (LODs) between electron capture negative ionization (ECNI) and electron ionization (EI) mass spectrometry (MS) were found to be insignificant for a wide range of aldehydes derivatized with $o-(2,3,4,5,6-$ pentafluorobenzyl)-hydroxylamine hydrochloride. Comparison of the two ionization methods based on LOD confidence intervals revealed that a traditional presentation of the LOD or limit of quantitation (LOQ) as a single value may over/underestimate the significance of obtained results. LODs were between 20 and $150 \mathrm{pg}$ injected for the majority of tested derivatized carbonyls using both ionization methods. ECNI-MS improved LODs by 10- to 20 -fold only for two derivatized aldehydes, 4-hydroxybenzaldehyde and 5-(hydroxymethyl)furfural. Selectivity of ECNI did not appear to be beneficial when analyzing a wood smoke particulate matter (WS-PM) extract, possibly because the majority of interferences were removed during sample preparation (i.e., liquid-liquid extraction). The impact of four different data acquisition modes of transmission quadrupole (TQ)-MS on LODs and their precisions was also investigated. As expected, LODs in the selected ion monitoring (SIM) were $\sim$ two to four times lower than those obtained using total ion current (TIC) mode. More importantly, TQ-MS in the selected ion-total ion (SITI) mode (i.e., acquiring SIM and TIC data in a single analysis) provided signal-to-noise ratios and precisions, which were comparable to SIM alone. (J Am Soc Mass Spectrom 2010, 21, 592-602) (c) 2010 American Society for Mass Spectrometry

A ldehydes, as products of the lipid peroxidation reactions and various atmospheric processes, are often detected in biological and environmental matrices [1-15]. Derivatization of aldehydes is typically employed before the analysis to improve chromatographic separation and detection limits [1-15].

One of the suitable derivatization agents for aldehydes, recommended by the U.S. Environmental Protection Agency (EPA), is o-(2,3,4,5,6-pentafluorobenzyl)hydroxylamine hydrochloride (PFBHA) [11, 16]. Reaction of aldehydes with PFBHA provides thermally stable and volatile oximes amenable to gas chromatographic (GC) analysis [16, 17]. Mass spectrometry (MS), using either electron ionization (EI) or electron capture negative ionization (ECNI), is often employed for detection of PFBHA-aldehydes.

Use of selective and sensitive ECNI is enabled due to the strong electron-withdrawing effect of five fluorine atoms in the molecule of derivatized aldehyde (PFBHA-

Address reprint requests to Dr. A. Kubátová, Department of Chemistry, University of North Dakota, 151 Cornell Street Stop 9024, Grand Forks, ND 58202, USA. E-mail: akubatova@chem.und.edu aldehyde). In contrast to high-energy (i.e., hard ionization) EI-MS, soft ionization (e.g., ECNI) does not typically induce extensive fragmentation of a molecular ion. A negative-charge molecular ion (i.e., $[\mathrm{M}]^{-\bullet}$ ) may appear with a high abundance in an ECNI spectrum facilitating the analyte identification as well as increasing sensitivity [18]. It was observed, however, that the fragmentation of PFBHA-aldehyde negative-charge molecular ions (namely $\mathrm{C}_{6}-\mathrm{C}_{10}$ linear aldehydes, glyoxal, methylglyoxal, and benzaldehyde) in ECNI was as extensive as that of positive-charge molecular ions in EI, perhaps due to the dominating dissociative electron capture mechanism of ionization [1, 11]. As a consequence, the identification of unknown PFBHAaldehydes is not facilitated using ECNI. The main ions observed in both EI and ECNI mass spectra originated from the derivatization agent. EI mode showed patterns with a base peak of $m / z 181$, which is $\left[\mathrm{C}_{6} \mathrm{~F}_{5} \mathrm{CH}_{2}\right]^{+}$[1]. Similarly, the base peaks observed in the derivatized aldehyde ECNI mass spectra represented the ions of $\left[\mathrm{C}_{6} \mathrm{~F}_{4} \mathrm{CH}_{2} \mathrm{O}\right]^{-}(\mathrm{m} / \mathrm{z} 178),\left[\mathrm{C}_{6} \mathrm{~F}_{5} \mathrm{CH}_{2}\right]^{-}(\mathrm{m} / \mathrm{z} 181)$, and $\left[\mathrm{C}_{6} \mathrm{~F}_{5} \mathrm{CHO}\right]^{-}(\mathrm{m} / z$ 196) [1]. A significant [M - 20] ion (perhaps $[\mathrm{M}-\mathrm{HF}]^{-}$) was observed in the mass spectra 
of $\mathrm{C}_{3}-\mathrm{C}_{12}$ saturated and unsaturated aliphatic aldehydes and some aromatic aldehydes in ECNI spectra $[1,11]$.

Limits of detection (LODs) obtained using ECNI and EI have been previously compared for only a few PFBHA-aldehydes (linear saturated and unsaturated aldehydes, glyoxal, methylglyoxal, and benzaldehyde), which showed ECNI to provide slightly lower LODs than EI $[1,19]$. However, neither the method of precision nor the description of LOD calculation were reported [1, 7, 10, 19-22]. EI and ECNI experiments were performed using different instrumentation [1, 19]; therefore, it is not clear whether the ECNI signal-tonoise ratio (i.e., detectability) improved solely due to a lower LODs of ECNI for PFBHA-aldehydes.

In this work, we have evaluated and compared the ECNI and EI-MS based on LOD confidence intervals for 33 aldehydes derivatized with PFBHA. The evaluation was performed using two GC/MS instruments (3D quadrupole ion trap and transmission quadrupole) each equipped with interchangeable CI and EI sources. We have also investigated the performance of transmission quadrupole (TQ)-MS in four data acquisition modes.

\section{Experimental}

\section{Chemicals and Materials}

Standards of aldehydes were of $95 \%$ and higher purity (unless stated otherwise). Acetaldehyde, propanal, butanal, isobutanal, $n$-pentanal, $n$-hexanal, $n$-heptanal, $n$ octanal, n-nonanal, n-decanal, $n$-undecanal, $n$-dodecanal, acrolein, trans-2-pentenal, trans-2-hexenal, trans-2nonenal, trans,trans-2,4-nonadienal $(>85 \%)$, glyoxal $(40 \%$ wt. in water), methylglyoxal $(40 \%$ wt. in water), glutaraldehyde (50\% wt. in water), benzaldehyde, $o$-tolualdehyde, $m$-tolualdehyde, phenylacetaldehyde $(>90 \%)$, hydrocinnamaldehyde $(>90 \%)$, 2,5-dimethylbenzaldehyde, 2-furaldehyde, and 5(hydroxymethyl)furaldehyde were purchased from Sigma-Aldrich (Milwaukee, WI, USA). Formaldehyde (36.6\% wt. in water), crotonal, 2-hydroxybenzaldehyde, 4-hydroxybenzaldehyde, and $p$-anisaldehyde were purchased from Chem Service (West Chester, PA, USA). Benzaldehyde- $d_{6}$ (99.6 atom \% D) and butanal-2,2- $d_{2}$ (99.4 atom \% D) were purchased from CDN Isotopes (Pointe-Claire, QC, Canada). PFBHA (>99\%) was purchased from Alfa-Aesar (Ward Hill, MA, USA). Ultra high purity helium (99.9995\%) and methane (99.999\%) were purchased from Airgas (Grand Forks, ND, USA). Methanol (LC/MS optima grade) and dichloromethane (DCM) of GC/MS quality were purchased from Fisher Scientific (Pittsburg, PA, USA). Water was purified using a Direct-Q3 water purification system with an incorporated dual wavelength UV lamp (Millipore, Billerica, MA, USA) for low total organic carbon content (the manufacturer's claimed purity is less than $5 \mathrm{ng} \mathrm{g}^{-1}$ ).

\section{PFBHA-Aldehydes Preparation}

Individual standard stock solutions of aldehydes were prepared in methanol at a concentration of $\sim 20 \mathrm{mg}$ $\mathrm{mL}^{-1}$ and stored at $-18^{\circ} \mathrm{C}$. The derivatization method has been described elsewhere and was adopted from the EPA 556 method [16, 23]. Triplicates of PFBHAderivatized calibration standards $\left(10-600 \mu \mathrm{g} \mathrm{L}^{-1}\right)$ were prepared by spiking the appropriate amounts of aldehyde mixture into purified water. Two internal standards (IS), butanal- $d_{2}$ and benzaldehyde- $d_{6}$, were added to this solution in the final concentrations of $0.500 \mathrm{mg}$ $\mathrm{L}^{-1}$. PFBHA solution $\left(1.7 \times 10^{4} \mathrm{mg} \mathrm{L}^{-1}\right)$ was prepared fresh in purified water before the derivatization. A quantitative reaction of aldehydes was assured by adding PFBHA in excess (at least 10-fold based on molar ratios). To adjust the $\mathrm{pH}$ to 3.5, a few drops of an acid $\left(1 \times 10^{-3} \mathrm{~mol} \mathrm{~L}^{-1} \mathrm{H}_{2} \mathrm{SO}_{4}\right)$ or base $\left(1 \times 10^{-3} \mathrm{~mol} \mathrm{~L}^{-1}\right.$ $\mathrm{NaOH})$ were added. The final volume of the aqueous calibration standard solution was $5.0 \mathrm{~mL}$.

Solutions were set at room temperature in the dark for $12 \mathrm{~h}$ to complete the reaction [16]. To avoid potential interferences from the excess of PFBHA derivatization agent during the GC analysis, a few drops of concentrated sulfuric acid were added after the reaction. An extraction of PFBHA-aldehydes into $1.0 \mathrm{~mL}$ of DCM was then employed. The extraction was repeated three times [16]. The test-tube was hand-shaken for $1 \mathrm{~min}$ before withdrawing the DCM layer; the DCM fractions were combined (total volume was $3 \mathrm{~mL}$ ) and filtered through anhydrous $\mathrm{Na}_{2} \mathrm{SO}_{4}$ to remove residual water [16]. The resulting DCM solutions were concentrated under a gentle stream of nitrogen to $0.4 \mathrm{~mL}$ and analyzed using GC/MS.

Wood smoke particulate matter was extracted using hot pressurized water, which is described in detail elsewhere [23-25]. Aqueous extracts with aldehydes were prepared for GC/MS analysis as calibration standards.

\section{GC with ECNI- and EI-TQ-MS}

An Agilent 6890 GC coupled to Agilent 5975C inert XL EI/CI MSD (Agilent Technologies, Inc., Wilmington, DE, USA) and Gerstel MPS2 autosampler (Gerstel, Baltimore, MD, USA) were used. MSD ChemStation D.03.00.611 software was employed for the data acquisition and integration of chromatographic peak areas. The data obtained were further processed in Microsoft Office Excel.

The automated tune and $m / z$ calibration using the instrument's Autotune file were performed after every change of a source or at least once a week. No significant adjustments of EI or ECNI-MS parameters were observed among individual tunes. Pentafluorobenzylbutylamine (PFTBA) was used as a mass calibrant for the EI mode and perfluoro-5,8-dimethyl3,6,9-trioxydodecane (PFDTD) for the ECNI mode. The MS instrument was equilibrated overnight after 
Table 1. The parameters of analysis and quantitation employed for PFBHA-derivatized aldehydes including: quantitation and confirmation ions $(\mathrm{m} / \mathrm{z})$ used in EI and ECNI for both TQ-MS and QIT-MS, ISs employed for the quantitation of particular analytes, number of ions scanned, and scanning rates $\left(\mathrm{s}^{-1}\right)$ in SIM and SITI (applicable for TQ-MS only). Scanning rate in SITI mode is reduced due to the switching between TIC and SIM. The dwell time was $25 \mathrm{~ms}$ for all ions in SIM and SITI. SIM acquisition ions were the same as quantitation and confirmation ions

\begin{tabular}{|c|c|c|c|c|c|}
\hline \multirow[b]{2}{*}{$\begin{array}{l}\text { PFBHA derivatized } \\
\text { aldehyde }\end{array}$} & \multirow{2}{*}{$\begin{array}{l}\text { Molecular } \\
\text { weight of } \\
\text { derivative } \\
\left(\mathrm{g} \cdot \mathrm{mol}^{-1}\right)\end{array}$} & \multirow{2}{*}{$\begin{array}{c}\text { Internal } \\
\text { standard } \\
\text { used for } \\
\text { quantification }\end{array}$} & \multicolumn{3}{|c|}{ El-MS } \\
\hline & & & $\begin{array}{l}\text { Quantitation } \\
\text { ion } \mathrm{m} / \mathrm{z}\end{array}$ & $\begin{array}{l}\text { Confirmation } \\
\text { ion } m / z\end{array}$ & $\begin{array}{l}\text { Number of ion } \\
\text { ions scanned } \\
\text { in SIM group }\end{array}$ \\
\hline \multicolumn{6}{|l|}{ Aliphatic saturated aldehydes } \\
\hline Formaldehyde & 225 & Butanal- $\mathrm{d}_{2}$ & 181 & 195,225 & 4 \\
\hline Acetaldehyde & 239 & Butanal- $\mathrm{d}_{2}$ & 181 & 209,239 & 3 \\
\hline Propanal & 253 & Butanal- $\mathrm{d}_{2}$ & 181 & 223,236 & 3 \\
\hline Isobutanal & 267 & Butanal- $\mathrm{d}_{2}$ & 181 & 250 & 2 \\
\hline Butanal & 267 & Butanal- $\mathrm{d}_{2}$ & 239 & 226 & 4 \\
\hline Pentanal & 281 & Benzaldehyde- $d_{6}$ & 181 & 207,239 & 3 \\
\hline Hexanal & 295 & Benzaldehyde- $d_{6}$ & 181 & 239,295 & 3 \\
\hline Heptanal & 309 & Benzaldehyde- $d_{6}$ & 181 & 207,239 & 3 \\
\hline Octanal & 323 & Benzaldehyde- $d_{6}$ & 181 & 239,323 & 3 \\
\hline Nonanal & 337 & Benzaldehyde- $d_{6}$ & 181 & 239 & 2 \\
\hline Decanal & 351 & Benzaldehyde- $d_{6}$ & 181 & 239,351 & 3 \\
\hline Undecanal & 365 & Benzaldehyde- $d_{6}$ & 239 & 181,345 & 5 \\
\hline Dodecanal & 379 & Benzaldehyde- $d_{6}$ & 181 & 239 & 2 \\
\hline \multicolumn{6}{|l|}{ Aliphatic unsaturated aldehydes } \\
\hline Acrolein & 251 & Butanal- $d_{2}$ & 181 & 221,251 & 3 \\
\hline Crotonal & 265 & Butanal- $d_{2}$ & 181 & 195,250 & 3 \\
\hline trans-2-Pentanal & 279 & Benzaldehyde- $d_{6}$ & 181 & 250,279 & 3 \\
\hline trans-2-Hexenal & 293 & Benzaldehyde- $d_{6}$ & 181 & 250,293 & 3 \\
\hline trans-2-Nonenal & 335 & 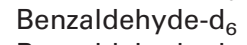 & 181 & 250,335 & 5 \\
\hline trans, trans-2,4-nonadienal & 333 & 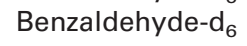 & 181 & 276,333 & 3 \\
\hline \multicolumn{6}{|l|}{ Aromatic aldehydes } \\
\hline Benzaldehyde & 301 & Benzaldehyde- $d_{6}$ & 301 & 271 & 4 \\
\hline m-Tolualdehyde & 315 & Benzaldehyde- $d_{6}$ & 181 & 91,315 & 4 \\
\hline o-Tolualdehyde & 315 & Benzaldehyde- $d_{6}$ & 181 & 91,315 & 4 \\
\hline 2,5-Dimethylbenzaldehyde & 329 & 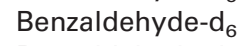 & 181 & 286,329 & 5 \\
\hline Phenylacetaldehyde & 315 & 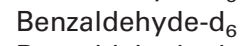 & 181 & 91,315 & 4 \\
\hline Hydrocinnamaldehyde & 329 & Benzaldehyde- $d_{6}$ & 181 & 271,329 & 5 \\
\hline p-Anisaldehyde & 331 & Benzaldehyde- $d_{6}$ & 331 & 181,288 & 5 \\
\hline 2-Hydroxybenzaldehyde & 317 & Benzaldehyde- $d_{6}$ & 181 & 300,317 & 3 \\
\hline 4-Hydroxybenzaldehyde & 317 & 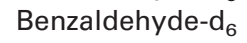 & 181 & 274,317 & 3 \\
\hline 2-Furfural & 291 & Benzaldehyde- $d_{6}$ & 291 & 248 & 3 \\
\hline 5-(Hydroxymethyl)furfural & 321 & Benzaldehyde- $d_{6}$ & 181 & 291,321 & 5 \\
\hline \multicolumn{6}{|l|}{ Dialdehydes } \\
\hline Glyoxal & 448 & Benzaldehyde- $d_{6}$ & 181 & 418,448 & 5 \\
\hline Methylglyoxal & 462 & Benzaldehyde- $d_{6}$ & 181 & 432,462 & 3 \\
\hline Glutaraldehyde & 490 & ${\text { Benzaldehyde- } d_{6}}$ & 181 & 293,490 & 3 \\
\hline
\end{tabular}

changing the EI to the CI source. The ion gauge pressure readings were $7.5 \times 10^{-6}$ Torr in EI and 1.7 $\times 10^{-4}$ Torr in ECNI.

Injections were performed in a splitless mode for 0.5 $\min$ at $250{ }^{\circ} \mathrm{C}$. Injection volume was $1 \mu \mathrm{L}$. The separation was performed on a $30-\mathrm{m}$ long, $0.25 \mathrm{~mm}$ i.d., 0.25 $\mu \mathrm{m}$ film thickness fused silica DB-5MS column (J and W Scientific, Folsom, CA, USA). A constant carrier gas flow rate of $1 \mathrm{~mL} \mathrm{~min}^{-1}$ was maintained during the analysis. The following temperature program was used: $50{ }^{\circ} \mathrm{C}$ held for $2 \mathrm{~min}$, followed by a $3^{\circ} \mathrm{C} \mathrm{min}^{-1}$ gradient to $230{ }^{\circ} \mathrm{C}$, then a $35^{\circ} \mathrm{C} \mathrm{min}{ }^{-1}$ gradient to $320^{\circ} \mathrm{C}$. The final temperature was held for $5 \mathrm{~min}$. The temperature of the
MS transfer line was kept at $280{ }^{\circ} \mathrm{C}$. The EI source temperature was $230^{\circ} \mathrm{C}$ and the ionization energy was 70 $\mathrm{eV}$. The CI source temperature in negative mode was evaluated within a range of $155-250{ }^{\circ} \mathrm{C}$. The optimal temperature was found to be $155^{\circ} \mathrm{C}$. The ECNI buffer gas (methane) was ionized using $230 \mathrm{eV}$; its flow was optimized within $0.5-2 \mathrm{~mL} \mathrm{~min}^{-1}$. The optimal flow rate was found to be $1.5 \mathrm{~mL} \mathrm{~min}{ }^{-1}$ (optimization is further discussed in the Results section).

To compare the sensitivity using EI and ECNI, data were acquired in the selected ion monitoring (SIM) mode using a unit mass resolution (i.e., denoted as a low-resolution in the acquisition software). 
Table 1. Continued

\begin{tabular}{|c|c|c|c|c|c|c|}
\hline \multicolumn{2}{|c|}{ EI-MS } & \multicolumn{5}{|c|}{$\mathrm{NCl}-\mathrm{MS}$} \\
\hline $\begin{array}{l}\text { Acquisition } \\
\text { rate in SIM } \\
\qquad\left(s^{-1}\right)\end{array}$ & $\begin{array}{c}\text { Acquisition } \\
\text { rate in SITI } \\
\left(\mathrm{s}^{-1}\right)\end{array}$ & $\begin{array}{l}\text { Quantitation } \\
\text { ion } \mathrm{m} / \mathrm{z}\end{array}$ & $\begin{array}{l}\text { Confirmation } \\
\text { ion } m / z\end{array}$ & $\begin{array}{l}\text { Number of ion } \\
\text { ions scanned } \\
\text { in SIM group }\end{array}$ & $\begin{array}{l}\text { Acquisition } \\
\text { rate in SIM } \\
\left(\mathrm{s}^{-1}\right)\end{array}$ & $\begin{array}{c}\text { Acquisition } \\
\text { rate in SITI } \\
\left(s^{-1}\right)\end{array}$ \\
\hline 6.20 & 3.97 & 181 & 178,225 & 3 & 8.10 & 4.87 \\
\hline 8.10 & 4.78 & 181 & 178,197 & 3 & 8.10 & 4.87 \\
\hline 8.10 & 4.78 & 181 & 178,233 & 3 & 8.10 & 4.87 \\
\hline 11.80 & 5.93 & 178 & 181,247 & 3 & 8.10 & 4.87 \\
\hline 6.20 & 4.02 & 247 & 220,267 & 6 & 4.20 & 3.07 \\
\hline 8.10 & 4.78 & 178 & 181,261 & 3 & 8.10 & 4.87 \\
\hline 8.10 & 4.78 & 178 & 181,275 & 3 & 8.10 & 4.87 \\
\hline 8.10 & 4.78 & 178 & 181,289 & 3 & 8.10 & 4.87 \\
\hline 8.10 & 4.74 & 178 & 276,303 & 3 & 8.10 & 4.87 \\
\hline 11.80 & 5.93 & 178 & 317,337 & 3 & 8.10 & 4.87 \\
\hline 8.10 & 4.74 & 178 & 196,331 & 3 & 8.10 & 4.87 \\
\hline 4.90 & 3.40 & 345 & 318 & 4 & 6.20 & 4.08 \\
\hline 11.80 & 5.93 & 178 & 196,359 & 3 & 8.10 & 4.87 \\
\hline 8.10 & 4.78 & 231 & 178, 201 & 4 & 6.20 & 4.08 \\
\hline 8.10 & 4.78 & 245 & 178,215 & 3 & 8.10 & 4.87 \\
\hline 8.10 & 4.78 & 259 & 178,229 & 3 & 8.10 & 4.87 \\
\hline 8.10 & 4.78 & 273 & 178,243 & 3 & 8.10 & 4.87 \\
\hline 4.90 & 3.40 & 178 & 315 & 5 & 4.90 & 3.45 \\
\hline 8.10 & 4.74 & 196 & 283,313 & 3 & 8.10 & 4.87 \\
\hline 6.20 & 4.02 & 281 & 251 & 4 & 6.20 & 4.08 \\
\hline 6.20 & 3.97 & 295 & 167,265 & 3 & 8.10 & 4.87 \\
\hline 6.20 & 3.97 & 295 & 167,265 & 3 & 8.10 & 4.87 \\
\hline 4.90 & 3.40 & 309 & 167,279 & 3 & 8.10 & 4.87 \\
\hline 6.20 & 3.97 & 178 & 204,268 & 3 & 8.10 & 4.87 \\
\hline 4.90 & 3.40 & 178 & 279,309 & 5 & 4.90 & 3.45 \\
\hline 4.90 & 3.40 & 311 & 178,281 & 4 & 6.20 & 4.08 \\
\hline 8.10 & 4.74 & 136 & 196,280 & 3 & 8.10 & 4.87 \\
\hline 8.10 & 4.74 & 297 & 178,267 & 3 & 8.10 & 4.87 \\
\hline 8.10 & 4.78 & 241 & 178,271 & 3 & 8.10 & 4.87 \\
\hline 4.90 & 3.40 & 271 & 196,255 & 3 & 8.10 & 4.87 \\
\hline 4.90 & 3.40 & 267 & 167,196 & 3 & 8.10 & 4.87 \\
\hline 8.10 & 4.74 & 281 & 167,196 & 3 & 8.10 & 4.87 \\
\hline 8.10 & 4.74 & 178 & 197,450 & 3 & 8.10 & 4.87 \\
\hline
\end{tabular}

To evaluate the sensitivity in various TQ-MS modes, data acquisition was performed in a total ion current (TIC) in the $m / z$ range of 50-600, SIM, and selected ion-total ion (SITI) mode (i.e., acquiring SIM and TIC data in a single analysis). Table 1 shows the acquisition ions (i.e., quantitation and confirmation ions) as well as data acquisition rates and a number of scanned ions in each SIM group (in SIM alone and in SITI). Analytes eluting close to each other from the GC column were acquired in one SIM group. Increased number of acquisition ions (e.g., five ions scanned for $p$-anisaldehyde in EI versus four ions in ECNI for the same compound) did not have a significant effect on LOD or precision. The fragmentation patterns of PFBHA-aldehydes obtained in both EI and ECNI resembled those previously published [1]. EI and ECNI spectra of selected analytes (to our knowledge not previously reported) are provided in the supplementary data, Figure S2, which can be found in the electronic version of this article.

In addition, TIC was performed using two scanning speeds. In this paper, one is referred as normal TIC (n-TIC), with a spectral acquisition rate of $\sim 2.66 \mathrm{~s}^{-1}$ in the $m / z$ range of 50-600 (providing $\sim 20-24$ data points across chromatographic peaks with half-widths of $\sim 7-8 \mathrm{~s}$ ), and the other is fast TIC (f-TIC), $\sim 13.24 \mathrm{~s}^{-1}$ in the same mass range (providing $\sim 100$ data points across chromatographic peaks with half-widths of $\sim 6-8 \mathrm{~s}$ ). The mass acquisition threshold was set to 50 counts for all methods (i.e., f-TIC, n-TIC, SIM, SITI). 


\section{GC with ECNI- and EI-QIT-MS}

A Trace GC equipped with Polaris-Q 291 3D quadrupole ion trap (QIT)-MS (further in the text referred as QIT-MS) with both EI and CI options and AS3000 autosampler (Thermo Fisher Scientific, Inc., Waltham, MA, USA) was used to confirm the trends of ECNI/ EI LODs for PFBHA-aldehydes observed using GC/ TQ-MS. Xcalibur 1.3 software was used for the data acquisition and integration of chromatographic peak areas. The data obtained were further processed in Microsoft Office Excel.

The automated tune and $\mathrm{m} / \mathrm{z}$ calibration using the instrument's Autotune file were performed after every change of the source or at least once a week. No significant adjustments of EI or ECNI-MS parameters were observed among individual tunes. PFTBA was used as a mass calibrant for both EI and ECNI modes. The CI ion volume was inserted into the MS ionization source for the ECNI experiment without breaking the vacuum. The instrument was equilibrated overnight. The ion gauge pressure readings were $2.0 \times 10^{-5}$ Torr in EI and $1.7 \times 10^{-4}$ Torr in ECNI.

Injection set-up, flow rate, column, oven temperature program, and transfer line temperature were the same as for GC/TQ-MS (see above). Ion source temperature during the EI experiment was $230{ }^{\circ} \mathrm{C}$ and the ionization energy was $70 \mathrm{eV}$. The ionization source temperature for ECNI was evaluated within a range of $155-250^{\circ} \mathrm{C}$. The optimal temperature was $155^{\circ} \mathrm{C}$. The ECNI buffer gas (methane) was ionized using $230 \mathrm{eV}$; its flow was optimized within $0.5-3 \mathrm{~mL}$

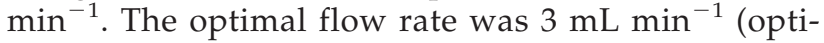
mization is further discussed in the Results and Discussion section). Data were acquired in the TIC mode (mass range from $\mathrm{m} / \mathrm{z} 50$ to 600) for both ECNI and EI (see Table 1 for quantitation and confirmation ions) experiments evaluating LODs of PFBHAderivatized aldehydes. QIT-MS acquisition parameters were set for both EI and ECNI as follows: number of microscans was 2, maximum ion time was $25 \mathrm{~ms}$, and automatic gain control was turned on. The average number of data points acquired across chromatographic peaks with half-widths of $\sim 6-8 \mathrm{~s}$ was $\sim 20-$ 24. The mass acquisition threshold was set to 50 counts.

\section{Statistical Data Evaluation}

LODs in $p g$ (a minimal mass injected enabling the analyte detection) and their confidence intervals for individual PFBHA-aldehydes were computed using data points from three calibration standard sets, which were within the linear range and one order of magnitude of suspected LOD [26]. A least-squares linear regression analysis using Microsoft Office Excel was performed to obtain calibration curve parameters. Average mass LODs $\left(m_{\mathrm{LOD}}\right)$ were calculated using eq 1 ,

$$
m_{L O D}=\frac{3.3 \times s_{Y}}{k}
$$

where $k$ is a slope of the calibration curve and $s_{\mathrm{y}}$ is the standard deviation of the linear regression residuals, which is obtained as a square root of residual mean square $\left(M S_{R E S}\right)$ representing the unbiased estimate of a calibration curve variance [27].

A confidence interval for $M S_{R E S}$ was obtained using eq 2 [27],

$$
\left\langle\frac{(n-c) M S_{R E S}}{\chi_{a / 2, n-c}^{2}} \leq M S_{R E S} \leq \frac{(n-c) M S_{R E S}}{\chi_{(1-a) / 2, n-c}^{2}}\right\rangle
$$

where $(n-c)$ represents a number of degrees of freedom ( $n$ is a number of data points, $c$ is a number of independent variables), $\chi^{2}$ is a chi-square distribution coefficient at a decision level $\alpha=0.05$ and degrees of freedom $(n-c)$, where $c=2$. The square root of upper and lower $M S_{R E S}$ gives the $s_{y}$ critical limits.

The confidence interval for $k$ was obtained using eq 3 [27],

$$
\left\langle k-t_{a / 2, n-c} s_{k} \leq k \leq k+t_{a / 2, n-c} s_{k}\right\rangle
$$

where $t$ is a coefficient of Student's $t$-distribution at a decision level $\alpha=0.05$ and degrees of freedom $(n-c)$, where $c=2, s_{\mathrm{k}}$ is a standard error of $k$ obtained from the regression analysis.

The uncertainty of $m_{\mathrm{LOD}}$ was estimated by propagation of errors eq 4 [assuming $m_{\mathrm{LOD}}$ as a function of $s_{\mathrm{y}}$ and $k($ eq 1$)$ ].

$$
d m_{L O D}\left(s_{Y}, k\right)=\sqrt{\left(\frac{\partial\left(3.3 s_{Y} / k\right)}{\partial s_{Y}} d s_{Y}\right)^{2}+\left(\frac{\partial\left(3.3 s_{Y} / k\right)}{\partial k} d k\right)^{2}}
$$

Solving and simplifying eq 4 assuming infinitesimal changes of $s_{\mathrm{y}}$ and $k$ (i.e., $\mathrm{d} s_{\mathrm{y}}=\Delta s_{\mathrm{y}}$ and $\mathrm{d} k=$ $\Delta k$ ) provided the uncertainty of $m_{\mathrm{LOD}}$ described in eq 5 .

$$
\Delta m_{\mathrm{LOD}}\left(s_{Y}, k\right)=\sqrt{\left(\frac{(3.3)}{k}\right)^{2} \Delta s_{Y}{ }^{2}+\left(\frac{-3.3 s_{Y}}{k^{2}}\right)^{2} \Delta k^{2}}
$$

Then the true value of $m_{\mathrm{LOD}}$ lies within the $95 \%$ confidence interval (eq 6).

$$
\left\langle m_{L O D}-\left(\Delta m_{L O D} / 2\right) \leq m_{L O D} \leq m_{L O D}+\left(\Delta m_{L O D} / 2\right)\right\rangle
$$

This confidence interval may be directly associated with the LOD precision. Calculations are graphically depicted 
in Figure 1a and b, (calibration curve parameters for all compounds and methods are provided in Table S1).

Finally, the hypothesis whether the average LODs obtained using EI and ECNI were different for a group of derivatized aldehydes (saturated and unsaturated aliphatic, aromatic aldehydes and dialdehydes) was tested using a two-tail paired $t$-test. Results were expressed as a probability (i.e., $P$ value). LODs obtained

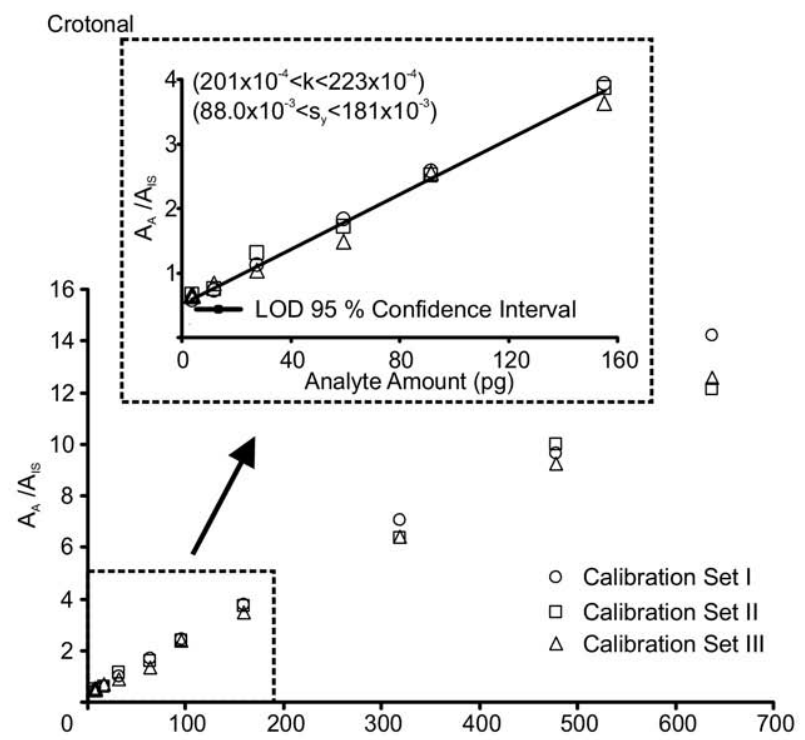

(a)

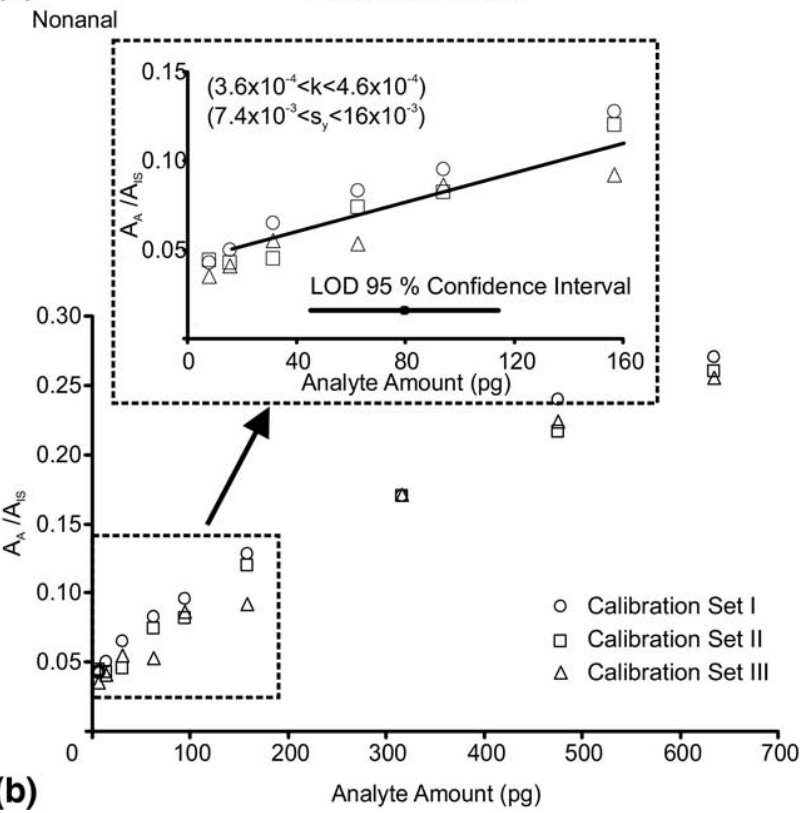

Figure 1. The effect of reproducibility in individual calibration levels on the LOD and its confidence interval for (a) crotonal and (b) nonanal, the width of $95 \%$ confidence interval for crotonal was 11-26 pg, whereas nonanal interval was broader, 47-115 pg, due to the low reproducibility in individual calibration levels (tabulated LOD intervals for all aldehydes and modes of acquisition are provided in supplementary Table S2). Data were acquired using ECNI-TQ-MS. Insets show data points, which were used for statistical calculations ( $\mathrm{s}_{\mathrm{y}}, \mathrm{k}$, and LOD confidence interval). $\mathrm{A}_{\mathrm{A}} /$ $\mathrm{A}_{\mathrm{IS}}$ is abundance of analyte/abundance of internal standard. for EI and ECNI were considered non-different if $P>$ 0.05 (with 95\% confidence).

\section{Results and Discussion}

\section{Optimization of ECNI Parameters}

The ECNI parameters (buffer gas flow and source ionization temperature) were optimized for both GC/ ECNI-QIT-MS and GC/ECNI-TQ-MS systems before evaluating the detection sensitivity.

The optimum ion source temperature of $155{ }^{\circ} \mathrm{C}$ for both MS instruments corresponded to the general manufacturers' recommendations. Increased ion source temperature $\left(175-250^{\circ} \mathrm{C}\right)$ negatively affected responses of quantitation ions (listed in Table 1) due to the extensive fragmentation at higher source temperatures (Figure S1 demonstrates the decrease of $\mathrm{m} / \mathrm{z} 178$ response for derivatized heptanal with the increasing ion source temperature).

Unlike the temperature, the optimal flow rate of buffer gas (methane) was different for GC/ECNIQIT-MS and GC/ECNI-TQ-MS. Responses of quantitation ions increased linearly with increasing flow rate up to $1.5 \mathrm{~mL} \mathrm{~min}^{-1}$ for GC/ECNI-TQ-MS. A signal drop for all PFBHA-aldehydes was observed when the buffer gas flow rate was above $1.5 \mathrm{~mL} \mathrm{~min}^{-1}$. By contrast, the analyte responses increased linearly with increasing flow rate up to $3 \mathrm{~mL} \mathrm{~min}^{-1}$ for GC/ECNI-QIT-MS. A buffer gas flow rate of $1.5 \mathrm{~mL} \mathrm{~min}^{-1}$ and $3.0 \mathrm{~mL} \mathrm{~min}^{-1}$ was selected as optimal for GC/ECNI-TQ-MS and GC/ECNI-QIT-MS, respectively.

A finding that optimal buffer gas flow rates vary for different instruments (i.e., manufacturers) implies that the design of ion source may affect the ECNI efficiency; therefore, optimization of ECNI parameters may be a crucial step in trace concentration analysis.

\section{Minimum Detectability Using ECNI and EI}

The minimum detectability of ECNI and EI was evaluated using LODs of PFBHA-aldehydes expressed as $95 \%$ confidence intervals (calculation described in the experimental section). This approach allows for evaluating the LOD or LOQ precision, which is otherwise neglected when LODs/LOQs are reported as single values. The effect of data scattering at individual calibration levels on the LOD precision is graphically depicted in Figure 1a and b. A broad confidence interval for nonanal (Figure 1b), compared with crotonal (Figure 1a), was caused by decreased reproducibility at low calibration levels.

Absence of peaks representing negative-charge molecular ions in spectra obtained using ECNI (due to the dissociative electron capture ionization mechanism) perhaps contributed to the LOD being similar for EI and ECNI-MS for the majority of derivatized aldehydes employing both GC/TQ-MS (Figure 2) and GC/ QIT-MS (Figure 3). 


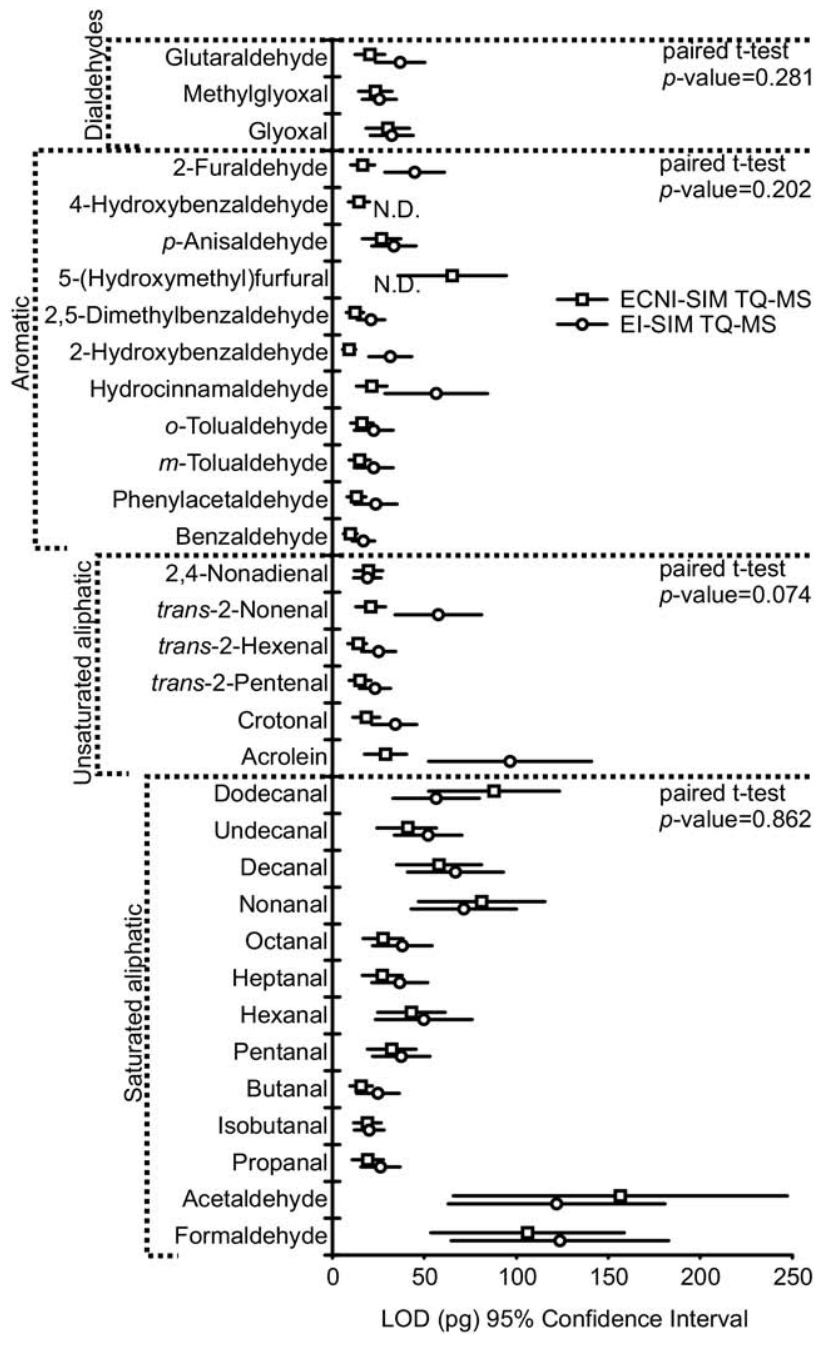

Figure 2. Comparison of LODs in $p g$ (graphically depicted as circles and squares) with their 95\% confidence interval (graphically depicted as lines) for EI-SIM-TQ-MS (open circle) and ECNI-SIM-TQ-MS (open square). Tabulated LOD intervals for all aldehydes and modes of acquisition are provided in the supplementary Table S2. Confidence intervals may be directly related to a method of precision at low detection levels. Two-tail paired $t$-test $P$ values show whether there is a significant difference in LODs between ECNI-SIM-TQ-MS and EI-SIM-TQ-MS for a group of aldehydes (saturated and unsaturated aliphatic, aromatic, and dialdehydes), the $P$ value $<0.05$ signifies that methods are different. N.D. $=$ not detected, within the tested range of 50-600 pg.

A small improvement (2-3 times) was observed for derivatized alkenals using ECNI-TQ-MS when evaluating the average LOD values (depicted as squares in Figure 2). However, based on the paired $t$-test, the two ionization methods (i.e., ECNI and EI) were not statistically different $(P$ value $=0.074)$ with $95 \%$ confidence (Figure 2). This trend was confirmed by QIT-MS analysis of derivatized alkenals where LODs obtained using ECNI and EI were nearly identical (Figure 3). Apparently only LOD precisions were slightly improved using ECNI-TQ-MS but average LODs remained similar to EI (Figure 2 and Figure 3).

The only significant improvement in LODs using ECNI (for both TQ-MS and QIT-MS) was observed for two PFBHA-derivatized hydroxylated aromatic aldehydes, 4-hydroxybenzaldehyde and (5-hydroxymethyl) furfural. Their LODs were in the same range (20-150 pg) as for other aldehydes using ECNI. However in EI, they were undetected or provided a weak response within the tested concentration range $\sim 10-600$ pg (Figure 2 and Figure 3). In contrast to 4-hydroxybenzaldehyde, 2hydroxybenzaldehyde was detected with comparable LODs in both ionization modes (Figure 2 and Figure 3). This suggests that the position of a functional group $(-\mathrm{OH})$ in a molecule may affect the EI efficiency.

In summary, the individual data acquisition methods mainly affected the precision of LOD but not the

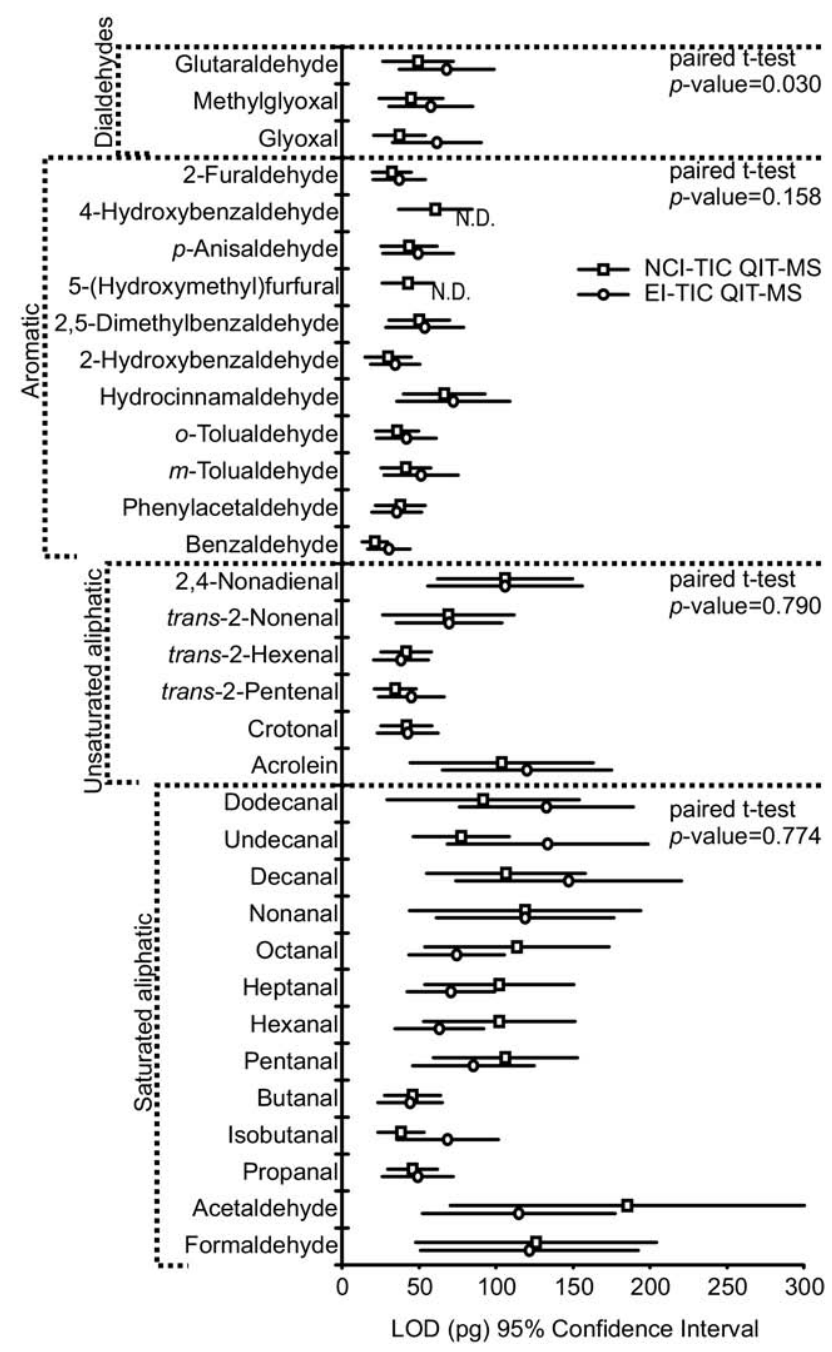

Figure 3. Comparison of LODs in $p g$ (graphically depicted as circles and squares) with their 95\% confidence interval (graphically depicted as lines) for EI-TIC-QIT-MS (open circle) and ECNI-TIC-QIT-MS (open square). Tabulated LOD intervals for all aldehydes and modes of acquisition are provided in the supplementary Table S2. Confidence intervals may be directly related to a method precision at low detection levels. Two-tail paired $t$-test $P$ value shows whether there is a significant difference in sensitivity between ECNI-TIC-QIT-MS and EI-TIC-QIT-MS for a group of aldehydes (saturated and unsaturated aliphatic, aromatic, and dialdehydes), the lower $P$ value the greater probability that methods are different. N.D. $=$ not detected within the tested range of $50-600 \mathrm{pg}$. 
detectability itself. It appeared that the LOD confidence intervals were a more appropriate representation of results than single LOD values, especially when the differences between two methods were relatively small. Consequently, comparing two techniques based only on single LOD values may over/underestimate the significance of obtained results.

\section{Selectivity of ECNI Versus EI in Analysis of Wood Smoke Particulate Matter Extract}

To investigate whether the selective ECNI may reduce/ eliminate background noise from complex-matrices, the extract of wood smoke particulate matter (WS-PM) was analyzed using GC/MS using both ECNI and EI (Figure $4 a-c)$.

As expected, chromatographic peaks representing methoxyphenols, polycyclic aromatic hydrocarbons (phenanthrene, fluoranthene), esters of carboxylic acids and some other compound types were observed in the EI chromatogram (Figure 4a) but not detected using ECNI (Figure 4b), which does not efficiently ionize these compounds. However, the overall chromatogram "baseline noise" of quantitation ions (listed in Table 1) was not improved in ECNI. Both ionization techniques provided similar signal-to-noise ratio for PFBHAaldehydes detected in WS-PM extract.

Based on the comparison of EI (Figure 4a) and ECNI (Figure $4 \mathrm{~b}$ ) chromatograms of derivatized and underivatized WS-PM extract (Figure 4c), it appeared that the majority of interferences were removed during sample preparation (including PFBHA derivatization and LLE).

\section{Effects of Data Acquisition Modes on LODs and Their Precisions}

Results discussed in the section "Minimum Detectability Using ECNI and EI" demonstrated that QIT-MS operated in TIC mode provided similar average LODs (in both EI and ECNI) for derivatized aromatic aldehydes and only slightly higher LODs for some aliphatic aldehydes than those obtained using TQ-MS in SIM (Figures 2, 3, and Table S2). The use of QIT-MS may thus be preferred in some cases (e.g., a complete characterization of sample is desired apart from the quantitation of targeted analytes) over SIM in TQ-MS because it provides full spectral information.

Recent advancements in quadrupole analyzer technologies (i.e., shortening the settling times between ion-scans and improving signal stability) have enabled additional data acquisition modes (besides TIC and SIM). New acquisition modes include SITI (i.e., acquiring SIM and TIC data in a single run) and fast scanning TIC (f-TIC). Due to these developments, TQ-MS may offer similar performance as QIT-MS.

We evaluated the efficiency of TQ-MS data acquisition modes including f-TIC, n-TIC, SITI, and SIM using LODs (i.e., signal-to-noise ratio) and precisions as cri-
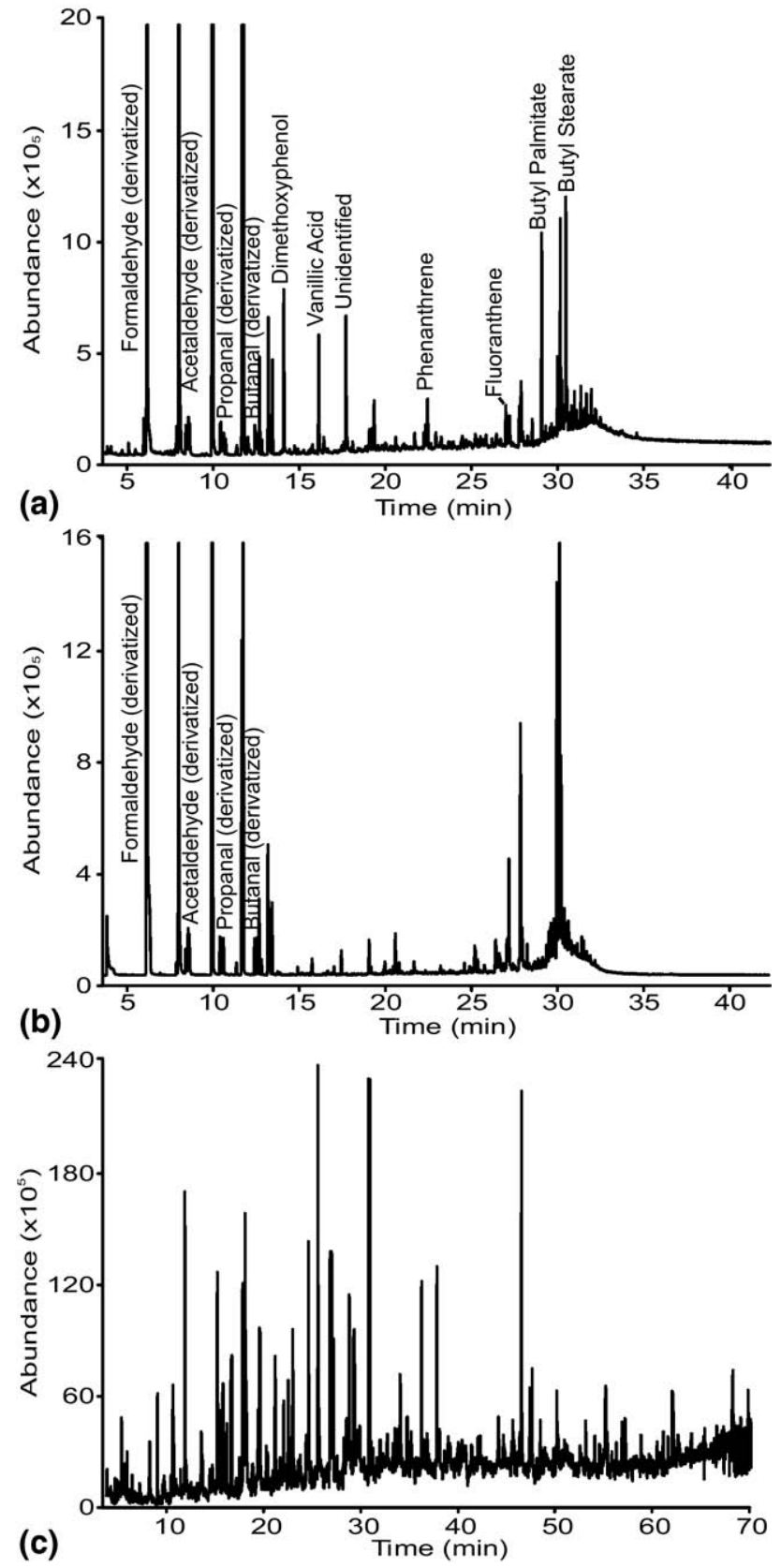

Figure 4. The chromatographic analysis of the wood smoke particulate matter extract obtained (a) after PFBHA derivatization using EI-TIC-TQ-MS, (b) after PFBHA derivatization using ECNI(TIC)-TQ-MS, and (c) using EI-(TIC)-TQ-MS (no derivatization applied), no aldehydes could be identified in this chromatogram due to the insufficient chromatographic resolution as well as strong interfering signal of other analytes.

teria (Figure 5 and Figure 6). Average LODs obtained by both n-TIC and f-TIC were not statistically different for the majority of analytes (Figure 5). For several compounds, including derivatized acrolein, trans-2nonenal, trans,trans-2,4-nonadienal, and dodecanal data acquisition in f-TIC resulted in a higher LOD than in n-TIC. The precision of LODs was also low when using f-TIC (Figure 5). Chromatographic peaks at low concentration levels acquired using f-TIC were noisy and 


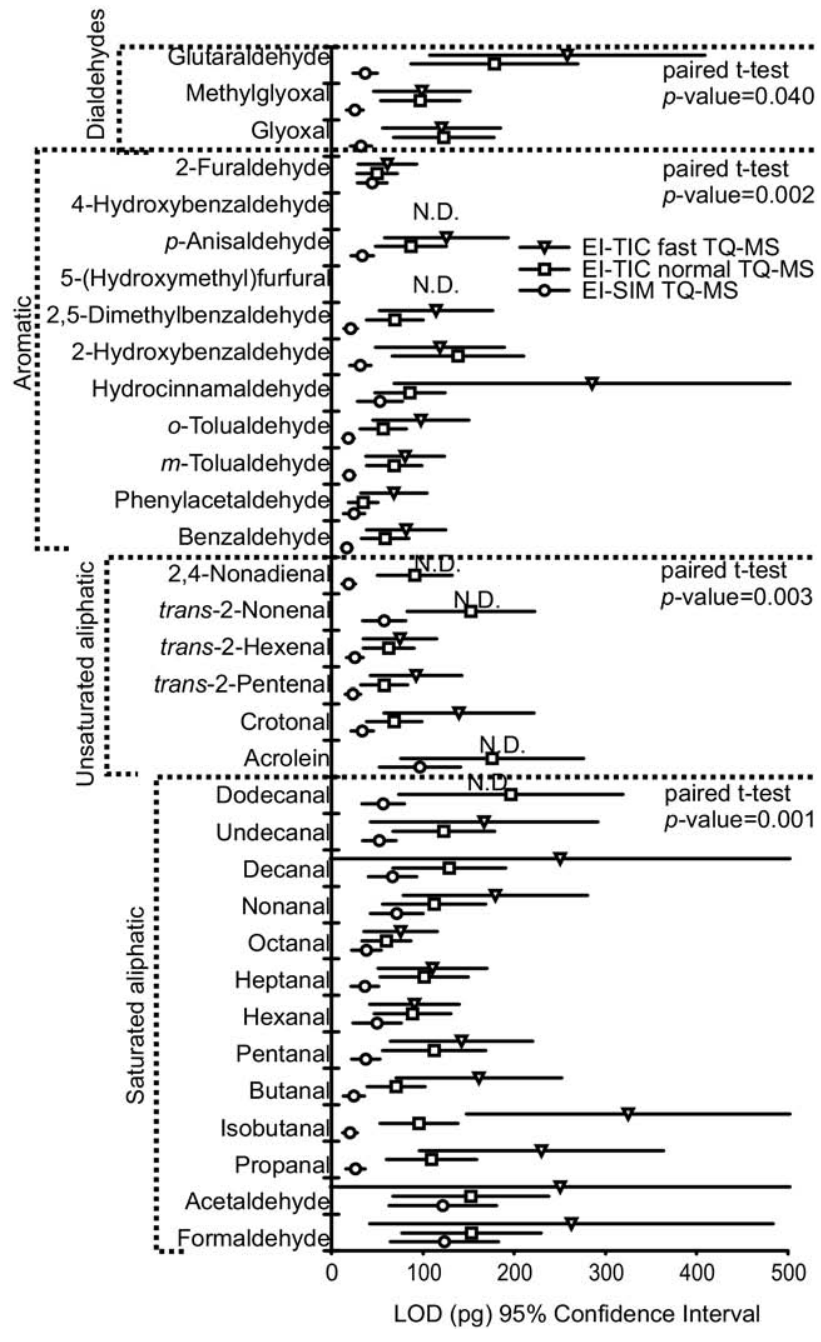

Figure 5. Comparison of LODs in $p g$ (graphically depicted as circles, squares, and triangles) with their $95 \%$ confidence interval (graphically depicted as lines) for EI-SIM-TQ-MS (open circle), EI-(n-TIC)-TQ-MS (open square), and EI-(f-TIC)-TQ-MS (inverted open triangle). Tabulated LOD intervals for all aldehydes and modes of acquisition are provided in the supplementary Table S2. Confidence intervals may be directly related to a method precision at low detection levels. Two-tail paired $t$-test $P$ value shows whether there is a significant difference in sensitivity between EI-(n-TIC)-TQ-MS and EI-(SIM)-TQ-MS for a group of aldehydes (saturated and unsaturated aliphatic, aromatic, and dialdehydes), the lower $P$ value the greater probability that methods are different. N.D. $=$ not detected within the tested range of 50-600 pg.

distorted when compared with n-TIC (Figure 6a and b). Even though data smoothing was applied, peak areas were irreproducible resulting in a large data variability and lower f-TIC detectability for some compounds.

A large LOD variability was observed for lowmolecular weight derivatized formaldehyde, acetaldehyde, and acrolein using all TQ-MS acquisition modes as well as QIT-MS in TIC (Figures 2, 3, and 5). This was, however, attributed to their random evaporative losses during a controlled sample evaporation, which often must be employed to re-concentrate trace amounts of aldehydes in environmental samples [23]. A suitable
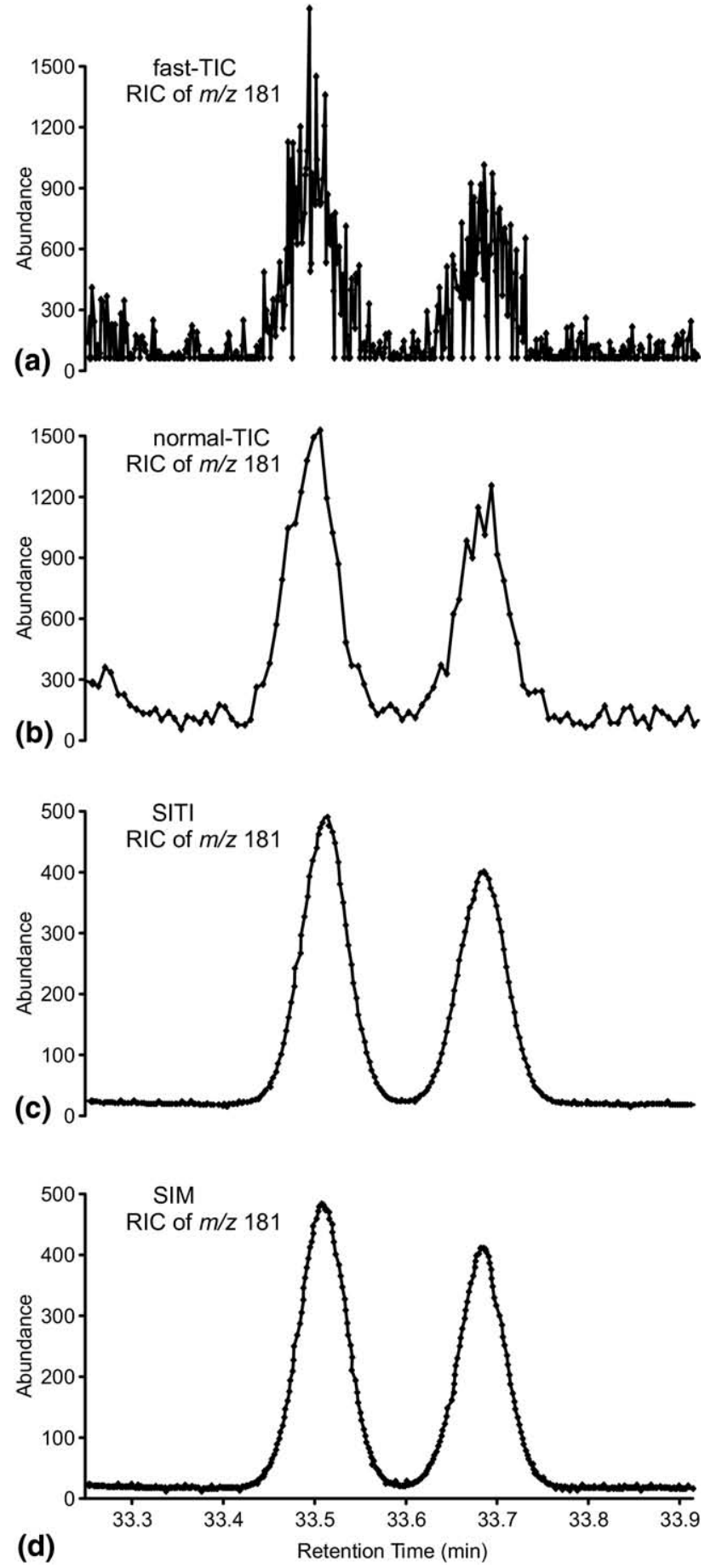

Figure 6. The reconstructed ion chromatograms (RIC) of $\mathrm{m} / \mathrm{z}$ of 181 of heptanal (syn/anti stereoisomers) acquired using (a) EI(f-TIC)-TQ-MS, (b) EI-(n-TIC)-TQ-MS, (c) EI-(SITI)-TQ-MS, where SIM signal was used, and (d) EI-(SIM)-TQ-MS. The peak halfwidths were $\sim 6 \mathrm{~s}$; f-TIC (a) provided $\sim 100$, n-TIC (b) $\sim 21$, SITI (c) $\sim 40$, and SIM (d) $\sim 70$ data points across the chromatographic peak. A low signal-to-noise ratio in (a) caused the decrease of detectability and reproducibility compared with (b). SITI (c) and SIM (d) had similar signal-to-noise ratio, thus the detectability and reproducibility (peak shape) of the two scanning methods were comparable. The amount of analyte was $150 \mathrm{pg}$, which was near LOD in TIC mode. 
way to account for this discrepancy, and thus improve the precision, would be to use the corresponding isotopically-labeled internal standards [23].

LODs and peak shapes (i.e., precision) in SITI (based on the SIM data) were not compromised compared with a simple SIM for all tested PFBHA-aldehydes (Figure 6c and d) despite the decreased number of data points acquired across the chromatographic peaks (e.g., from $\sim 70$ data points in SIM to $\sim 40$ in SITI for PFBHAheptanal, Figure $6 \mathrm{c}$ and d). TQ-MS in SITI mode may be equally or more efficient than QIT-MS in TIC.

As expected, the lowest LODs were obtained using SIM (or using SIM data from SITI mode). Average LODs were $\sim 2$ to 4 times lower than with n-TIC with significant improvement of the LOD precision as well (Figure 5 and Figure 6 ). The $P$ values obtained from a paired $t$-test of SIM and n-TIC (Figure 5) show that the two methods were statistically different in sensitivity comparing groups of derivatized aldehydes (saturated and unsaturated linear, aromatic aldehydes, and dialdehydes).

\section{Conclusions}

In contrast to general expectation, EI and ECNI-MS offered similar LODs for 31 of 33 tested PFBHAderivatized aldehydes. LODs were significantly decreased ( $\sim 10$ - to 20-fold) using ECNI only for two specific polar analytes, derivatized 4-hydroxybenzaldehyde and (5-hydroxymethyl)furfural. Assuming comparable LODs, EI may be suggested as a more suitable technique than ECNI due to the availability of EI-MS libraries, and predictability of fragmentation patterns. This is an important consideration when, in addition to the quantitation of targeted compounds, the identification of unknown peaks is required.

Comparing ECNI and EI based on their single-LOD values, without any consideration for precision, may over/underestimate the statistical significance of the obtained results. To overcome this problem, we derived an approach allowing for calculation of LOD with its confidence interval from the calibration curve. The statistical calculation also has a large potential for determining the limit of quantitation (LOQ) confidence intervals upon the proper modification of eqs 1,4 , and 5. Obtaining the LOQ confidence intervals may be critical when establishing/employing methods for quality control of regulated substances in various industries.

Finally, it was confirmed that signal-to-noise ratios and precisions for PFBHA-aldehydes in the SITI mode (acquisition of SIM and TIC data in a single analysis) were the same as in the SIM mode alone. TQ-MS in SITI was thus demonstrated to be a good alternative to QIT-MS in TIC for a sensitive analysis of complex samples.

\section{Acknowledgments}

The authors thank Dr. E. Kozliak and D. Stahl for valuable comments while writing and editing this manuscript. This work was sponsored by the North Dakota EPSCoR through NSF grants ATM-0747349 and EPS-0814442, and through the DOE grant DE-FG02-06ER46292, and by the National Center for Research Resources (NCRR), a component of the National Institutes of Health (NIH), through the grant P20RR17699-05; DSM gratefully acknowledges support from NSF CBET 0651058. The contents of this article are solely the responsibility of the authors and do not necessarily represent the official views of NSF, DOE, and NIH.

\section{Appendix A Supplementary Material}

Supplementary material associated with this article may be found in the online version at doi:10.1016/ j.jasms.2009.12.009.

\section{References}

1. Le Lacheur, R. M.; Sonnenberg, L. B.; Singer, P. C.; Christman, R. F.; Charles, M. J. Identification of Carbonyl Compounds in Environmental Samples. Environ. Sci. Technol. 1993, 27, 2745-2753.

2. Culleré, L.; Cacho, J.; Ferreira, V. Analysis for Wine C5-C8 Aldehydes Through the Determination of Their O-(2,3,4,5,6-Pentafluorobenzyl) Oximes Formed Directly in the Solid Phase Extraction Cartridge. Anal. Chim. Acta 2004, 524, 201-206.

3. Liggio, J.; McLaren, R. An Optimized Method for the Determination of Volatile and Semivolatile Aldehydes and Ketones in Ambient Particulate Matter. Int. J. Environ. Anal. Chem. 2003, 83, 819-835.

4. Pacolay, B. D.; Ham, J. E.; Wells, J. R. Use of Solid-Phase Microextraction to Detect and Quantify Gas-Phase Dicarbonyls in Indoor Environments. J. Chromatogr. A 2006, 1131, 275-280.

5. Frankel, E. N.; Hu, M. L.; Tappel, A. L. Rapid Headspace Gas Chromatography of Hexanal as a Measure of Lipid Peroxidation in Biological Samples. Lipids 1989, 24, 976-981.

6. Spiteller, P.; Kern, W.; Reiner, J.; Spiteller, G. Aldehydic Lipid Peroxidation Products Derived from Linoleic Acid. Biochim. Biophys. Acta Mol. Cell. Biol. Lipids 2001, 1531, 188-208.

7. Selley, M. L. Determination of the Lipid Peroxidation Product (E)-4Hydroxy-2-Nonenal in Clinical Samples by Gas ChromatographyNegative-Ion Chemical Ionization Mass Spectrometry of the OPentafluorobenzyl Oxime. J. Chromatogr. B 1997, 691, 263-268.

8. Teixido, E.; Santos, F. J.; Puignou, L.; Galceran, M. T. Analysis of 5-Hydroxymethylfurfural in Foods by Gas Chromatography-Mass Spectrometry. J. Chromatogr. A 2006, 1135, 85-90.

9. Spiteller, G.; Kern, W.; Spiteller, P. Investigation of Aldehydic Lipid Peroxidation Products by Gas Chromatography-Mass Spectrometry. J. Chromatogr. A 1999, 843, 29-98.

10. Luo, X. P.; Yazdanpanah, M.; Bhooi, N.; Lehotay, D. C. Determination of Aldehydes and Other Lipid Peroxidation Products in Biological Samples by Gas Chromatography-Mass Spectrometry. Anal. Biochem. 1995, 228, 294-298.

11. Hsu, F. F.; Hazen, S. L.; Giblin, D.; Turk, J.; Heinecke, J. W.; Gross, M. L. Mass Spectrometric Analysis of Pentafluorobenzyl Oxime Derivatives of Reactive Biological Aldehydes. Int. J. Mass Spectrom. 1999, 185/ 186/187, 795-812.

12. Müller, K.; Haferkorn, S.; Grabmer, W.; Wisthaler, A.; Hansel, A. Kreuzwieser, J.; Cojocariu, C.; Rennenberg, H.; Herrmann, H. Biogenic Carbonyl Compounds Within and Above a Coniferous Forest in Germany. Atmos. Environ. 2006, 40, 81-91.

13. Rubio, M. A.; Zamorano, N.; Lissi, E.; Rojas, A.; Gutierrez, L.; Von Baer, D. Volatile Carbonylic Compounds in Downtown Santiago, Chile. Chemosphere 2006, 62, 1011-1020.

14. Jakober, C. A.; Charles, M. J.; Kleeman, M. J.; Green, P. G. LC-MS Analysis of Carbonyl Compounds and Their Occurrence in Diesel Emissions. Anal. Chem. 2006, 78, 5086-5093.

15. Nagy, K.; Pollreisz, F.; Takats, Z.; Vekey, K. Atmospheric Pressure Chemical Ionization Mass Spectrometry of Aldehydes in Biological Matrices. Rapid Commun. Mass Spectrom. 2004, 18, 2473-2478.

16. U.S. Environmental Protection Agency. Determination of Carbonyl Compounds in Drinking Water by Pentafluorobenzylhydroxylamine Derivatization and Capillary Gas Chromatography with Electron Capture Detection. Method 556. Washington, DC.

17. Destaillats, H.; Charles, M. J. Henry's Law Constants of CarbonylPentafluorobenzyl Hydroxylamine (PFHBA) Derivatives in Aqueous Solution. J. Chem. Eng. Data 2002, 47, 1481-1487. 
18. Bezabeh, D. Z.; Bamford, H. A.; Schantz, M. M.; Wise, S. A. Determination of Nitrated Polycyclic Aromatic Hydrocarbons in Diesel Particulate-Related Standard Reference Materials by Using Gas Chromatography/Mass Spectrometry with Negative Ion Chemical Ionization. Anal. Bioanal. Chem. 2003, 375, 381-388.

19. Sugaya, N.; Sakurai, K.; Nakagawa, T.; Onda, N.; Onodera, S.; Morita, M.; Tezuka, M. Development of a Headspace GC/MS Analysis for Carbonyl Compounds (Aldehydes and Ketones) in Household Products After Derivatization with o-(2,3,4,5,6-Pentafluorobenzyl)-Hydroxylamine. Anal. Sci. 2004, 20, 865-870.

20. Sugaya, N.; Nakagawa, T.; Sakurai, K.; Morita, M.; Onodera, S. Analysis of Aldehydes in Water by Head Space-GC/MS. J. Health Sci. 2001, 47, 21-27.

21. Wichard, T.; Poulet, S. A.; Pohnert, G. Determination and Quantification of a,b,g,d-Unsaturated Aldehydes as Pentafluorobenzyl-Oxime Derivates in Diatom Cultures and Natural Phytoplankton Populations: Application in Marine Field Studies. J. Chromatogr. B 2005, 814, 155-161.

22. Li, Z.; Jacobus, L. K.; Wuelfing, W. P.; Golden, M.; Martin, G. P.; Reed, R. A Detection and Quantification of Low-Molecular-Weight Aldehydes in
Pharmaceutical Excipients by Headspace Gas Chromatography. J. Chromatogr. A 2006, 1104, 1-10.

23. Beránek, J.; Kubátová, A. Evaluation of Solid-Phase Microextraction Methods for Determination of Trace Concentration Aldehydes in Aqueous Solution. J. Chromatogr. A 2008, 1209, 44-54.

24. Kubatova, A.; Steckler, T. S.; Gallagher, J. R.; Hawthorne, S. B.; Picklo, M. J. Toxicity of Wide-Range Polarity Fractions from Wood Smoke and Diesel Exhaust Particulate Obtained Using Hot Pressurized Water. Environ. Toxicol. Chem. 2004, 23, 2243-2250.

25. Hawthorne, S. B.; Kubatova, A. Hot (Subcritical) Water Extraction. In Comprehensive Analytical Chemistry, 1st ed.; Barcelo, D., Eds. Elsevier: New York, NY, 2002; pp 587-608.

26. Pawliszyn, J. Solid Phase Microextraction. In Comprehensive Analytical Chemistry; Barcelo, D., Ed. Elsevier: New York, NY, 2002; pp 389-478.

27. Montgomery, D. C.; Peck, E. A.; Vinning, G. G. Introduction to Linear Regression Analysis, 4th ed.; John Wiley and Sons: Hoboken, NJ, 2006; pp 25-145. 